\title{
Band Selection for Hyperspectral Images Using Non-Negativity Constraints
}

\author{
John Gruninger, Hoang Dothe \\ Spectral Sciences Inc., 4 Fourth Ave, Burlington, MA 01803 USA \\ Correspondence: John Gruninger, john@spectral.com \\ Manuscript communication: received 27 February 2013, accepted 15 March 2014
}

\begin{abstract}
This paper presents a new factorization technique for hyperspectral signal processing based on a constrained singular value decomposition (SVD) approach. Hyperpectral images typically have a large number of contiguous bands that are highly correlated. Likewise the field of view typically contains a limited number of materials and the spectra are also correlated. Only a selected number of bands, the extreme bands that include the dominant materials spectral signatures, are needed to express the data. Factorization can provide a means for interpretation and compression of the spectral data. Hyperspectral images are represented as non-negative matrices by graphic concatenation, with the pixels arranged into columns and each row corresponding to a spectral band. SVD and principal component analysis enjoy a broad range of applications, including, rank estimation, noise reduction, classification and compression, with the resulting singular vectors forming orthogonal basis sets for subspace projection techniques. A key property of non-negative matrices is that their columns/rows form non-negative cones, with any non-negative linear combination of the columns/rows belonging to the cone. Data sets of spectral images and time series reside in non-negative orthants and while subspaces spanned by SVD include all orthants, SVD projections can be constrained to the non-negative orthants. In this paper we utilize constraint sets that confine projections of SVD singular vectors to lie within the cones formed by the spectral data. The extreme vectors of the cone are found and these vectors form a basis for the factorization of the data. The approach is illustrated in an application to hyperspectral data of a mining area collected by an airborne sensor.
\end{abstract}

Keywords- SVD, non-negative factorization, convex cones, linear homogeneous inequalities, spectral images.

\section{INTRODUCTION}

Hyperspectral data consist of images collected at many spectral channels (or bands). Hyperspectral images can be converted into data matrices by lexigraphic concatenation, assigning each channel into a row and each pixel spectrum to a column. These data matrices are non-negative, i.e., all matrix elements are positive or zero. The singular value decomposition (SVD) provides the best rank approximation to a data matrix in the Frobenius norm, making it ideal for both noise reduction and for data compression. This feature often has been exploited to estimate the number of dominate material components present in spectral data. A data matrix, $\mathbf{A}$, of $L$ rows, $M$ columns and a rank or pseudorank of $N$ is represented by its SVD as

$$
\mathbf{A}=\mathbf{U} \boldsymbol{\Lambda} \mathbf{V}^{T}=\sum_{k=1}^{N} \mathbf{u}_{k} \lambda_{k} \mathbf{v}_{k}^{T},
$$

where $\mathbf{U}$ is an $L$ by $N$ matrix of orthonormal vectors, $\mathbf{u}_{k}$ the left singular vectors. $\mathbf{V}$ is an $M$ by $N$ matrix of orthonormal vectors and $\mathbf{v}_{k}$ the right singular vectors. The matrix $\Lambda$ is an $N$ by $N$ diagonal matrix of singular values, $\lambda_{k}$. Here the singular values are assumed to be in descending order, $\lambda_{1} \geq \lambda_{2} \geq \cdots \geq \lambda_{N}$.

\subsection{Pseudo-Rank}

If all singular values beyond the $N^{\text {th }}$ are zero the matrix is of rank $N$. More commonly a criterion is used to decide where to truncate the expansion under the assumption that the remaining terms correspond to noise. The truncated number $N$ is then called the pseudo-rank. In this way the SVD is used for noise reduction, with the assumption that the remaining singular vectors only contain noise. The pseudo-rank has also been used as an estimate of the number of unique bands and the number of unique materials represented in a spectral data set, making it an important number to obtain for interpretation. Assuming that there are $N$ linear independent vectors implies there are $N$ unique materials and $N$ unique bands, thus interpretation can be achieved via a linear mixing model where a basis of material spectral or band images can be used in a constrained least squared process to factor the spectral matrix.

Perhaps the most challenging aspect of the problem is the determination of the most appropriate value for the pseudo-rank. The divide between singular vectors containing useful information and those containing noise is grey. Variations in illumination and material reflectance at a minimum affect the intensity of a materials spectrum. Together with multiple scattering of light from the ground surface and the atmosphere, materials may need to be modeled with more than one spectrum. Most of the energy of an SVD expansion results from the dominate materials in an image while the spectral variations of materials or additional rare materials can contribute at levels within the noise [1]. 


\subsection{Non-Negative Rank}

Recently additional approaches to estimating [2, 3] the pseudo-rank have been developed that utilize the non-negativity of the column mean of a non-negative matrix. These "virtual" dimension methods are being used regularly as a basis for estimating the appropriate pseudo-rank.

All of the efforts have been based on the assumption that in the absence of noise, there is a one to one correspondence between true rank and the number of abundant and rare components, that is, all of the components form a linearly independent set. While linear independence of a set of vectors requires that no vector of the set be represented as a linear combination of the remaining vectors of the set, non-negative linear independence of a set of non-negative vectors requires that no vector of the set can be represented as a non-negative linear combination of the remaining vectors. The appropriate useful quantity is thus the non-negative rank. The non-negative rank does not necessarily coincide with the rank. The concept and determination of non-negative rank is still an active subject of research $[4,5]$. Its value is bounded by the rank of the matrix, $N$, and the smaller of the row/column dimensions of the matrix. Unfortunately there are no algorithms that predict its value [6].

We introduce in this paper a cone model for nonnegative row or column vectors of a spectral matrix and use it to place constraints on projections of the singular vectors [7]. The resulting projections can be processed to extract extreme vectors of the cone, the number of which can exceed the rank of the constraint matrix and the data matrix. Once the extreme vectors have been identified in the data, their contributions can be determined by a constrained least squares fitting procedure, or by an oblique projection technique. The approach is outlined in Section 2 and demonstrated in Section 3. Conclusions are drawn in Section 4.

\section{Approach}

\subsection{Non-Redundant Constraints and Extreme Vectors}

It is useful to introduce the cone concept for identifying extreme vectors in spectral matrices. Spectral matrices are non-negative. In the following, an approach is to determine extreme vectors in spectral matrices is presented, based on identifying non-redundant constraints that appear in enforcing non-negative linear combination requirements for cone vectors. The column cone (rows) of a non-negative matrix $\mathbf{A}$ is formed from all non-negative combinations of its columns (rows). By definition, all vectors, $\mathbf{a}$, of the column cone satisfy

$$
\mathbf{a}=\mathbf{A p}, \quad \text { for all } \mathbf{p} \geq 0 .
$$

Extreme vectors (rows) cannot be represented by a positive linear combination of other vectors (rows) in the data. Non-extreme vectors (rows) can be modeled by a positive linear combination of extreme vectors (rows). The extreme vectors of the column cone will have all components of $\mathbf{p}$ equal to zero except for its own component which will be unity. This constraint condition can be applied directly to the singular vectors.

The approach is to find appropriate projections of the singular vectors determined by singular value decomposition. We work directly with SVD together with constraints that confine the singular vector projections to the cones formed the columns and rows of the matrix. The constraint that singular vector projections, be restricted to the cone are formed by using Equations (1) and (2) as

$$
\mathbf{a}=\mathbf{U z}=\mathbf{A V} \Lambda^{-1} \mathbf{z}=\mathbf{A p},
$$

and thus the vectors a can be projected from the left singular vectors and the constraint relation for the projection coefficients, $\mathbf{z}$, is

$$
\mathrm{Gz} \geq \mathbf{0},
$$

with

$$
\mathbf{G}=\mathbf{G} \Lambda^{-1}
$$

In a totally analogous way row cones can be defined via the transpose $\mathbf{A}^{T}$. The row cone is defined as all vectors, $\mathbf{b}$ satisfying

$$
\mathbf{b}=\mathbf{A}^{T} \mathbf{f}, \quad \text { for all } \mathbf{f} \geq \mathbf{0},
$$

and in terms of the right singular vectors as

$$
\mathbf{b}=\mathbf{V} \mathbf{w}=\mathbf{A}^{T} \mathbf{U} \boldsymbol{\Lambda}^{-1} \mathbf{w}=\mathbf{A}^{T} \mathbf{f} .
$$

The constraint relation for right singular projection coefficients $\mathbf{w}$ is thus

$$
\text { Hw } \geq 0,
$$

with

$$
\mathbf{H}=\mathbf{U} \boldsymbol{\Lambda}^{-1} \text {. }
$$

In the above, $\mathbf{G}$ and $\mathbf{H}$ are the constraint matrices (for column and row, respectively). We concentrate here on the row cone and row inequality constraints (Equation (8)), as the processing of the column cone is totally analogous. We first recall that dimension of $\mathbf{H}$ is $L \times N$, where $L$ is the number of spectral channels (bands) and $N$ is the matrix rank. The length of the column vector $\mathbf{w}$ is $N$, which is in general much smaller than L. The system of inequalities in Equation (8), formed by the rows of $\mathbf{H}$, therefore contains many redundancies, that need to be reduced to a set of nonredundant equations. The removal procedure will allow identification of extreme vectors.

From the products in Equations (7) and (8), we note that the constraint matrix $\mathbf{H}$ has one row for each column of $\mathbf{A}^{T}$, a band of the spectral matrix while the elements of each column of $\mathbf{H}$ are associated with a left singular vector $\left(\mathbf{u}_{k}\right)$ of $\mathbf{A}$ in order of decreasing singular value. The rows of $\mathbf{H}$ form a set of linear homogeneous inequalities, $\mathbf{H w} \geq \mathbf{0}$. The determination of the extreme vectors of $\mathbf{A}^{T}$ can then be accomplished by the reduction of the inequalities to a set of non-redundant inequalities. Redundant inequalities are those that can be removed from the set without changing the feasible region [8], and hence without changing the cone of $\mathbf{A}^{T}$. The removal of redundant constraints is an important 
process in linear and non-linear programming and there is continuing research in this field [9]. Reducing the inequalities redundancies amounts to finding the extreme (non-redundant) rows of $\mathbf{H}$. Once the nonredundant rows of $\mathbf{H}$ have been identified, the corresponding columns of $\mathbf{A}^{T}$ now identify the extreme band images of $\mathbf{A}$.

\subsection{Determining the Non-Redundant Constraints}

Our approach in this paper relies on elementary properties of inequalities. First, an inequality multiplied by a positive constant remains an inequality of the same sign, and second, a positive linear combination of inequalities also leads to an inequality of the same sign. Spectral images with many pixels containing the same material will have nearly identical spectra and nearly identical constraints. Thus the first property can be used to cluster rows of the constraint matrix, $\mathbf{H}$, into groups. All but one row of each group will be redundant. However, a more general approach is needed for rows that are far from group centers, those that correspond to spectrally mixed images.

We use a step-wise oblique projection technique to determine the non-redundant constraints. There are two tasks performed in each step. First a constraint row is identified as an extreme, second this row is projected from all remaining rows that are not identified as extreme. Our selection process of extremes is based on the particular features of the constraint matrix $\mathbf{H}$. There is a direct correspondence between the column indices and the singular vectors (see Equations (1) and (8)). The first column of $\mathbf{H}$ is proportional to the first left singular vector, $\mathbf{u}_{1}$ of $\mathbf{A}$, the second column to the second, $\mathbf{u}_{2}$ and finally the $N^{\text {th }}$ column and the $N^{\text {th }}$ $\mathbf{u}_{N}$. We can thus identify extreme vectors as those with small first coefficient relative to the remaining ones. We select the first extreme row as the row with the smallest relative contribution of the first coefficient. The remaining extreme rows are selected using a projection procedure decribed next.

\subsection{Oblique Projections}

The projection procedure removes the current identified extreme from the remaining rows by oblique projections. An oblique projection [10] is defined as

$$
\mathbf{P}=\mathbf{x}\left(\mathbf{y}^{T} \mathbf{x}\right)^{-1} \mathbf{y}^{T} .
$$

An oblique projection differs from an orthogonal projection

$$
\mathbf{O}=\mathbf{x}\left(\mathbf{x}^{T} \mathbf{x}\right)^{-1} \mathbf{x}^{T} .
$$

in that the vector $\mathbf{y}$ is not necessarily parallel with vector $\mathbf{x}$. The oblique projection chosen is as close to an orthogonal projection as possible while constraining the resulting current and previous projection coefficients to be non-negative. All previous projection coefficients are updated during the projection and either the projection is orthogonal or a previous coefficient is driven to zero and the projection is constrained to be oblique. After the projection we select the row with the largest residual in the $\ell_{\infty}$ norm. The choice of the $\ell_{\infty}$ norm results in the selection of the vector with the largest magnitude error in a coefficient associated with one of the singular vectors. Whenever the projection is oblique the active constraint removes a previous projection from the particular model [11].

The processing is illustrated by a simple example. Let the ith row of $\mathbf{H}, \mathbf{h}_{i}$ be the first extreme vector selected. Its projection is removed from all the remaining unselected $k^{\text {th }}$ rows $\mathbf{h}_{k}$ yielding

$$
\mathbf{h}_{k}^{(1)}=\mathbf{h}_{k}-\mathbf{h}_{i} \mathbf{c}_{i, k}
$$

where $\mathbf{c}_{i, k}$ are the projection coefficients, which are required to be positive. The projection will be orthogonal if $\mathbf{c}_{i, k} \geq 0$, otherwise, $\mathbf{c}_{i, k}$ is set to zero. Let the $j^{\text {th }}$ row be selected as the second extreme vector. It's removal from all the remaining kth rows is,

$$
\begin{gathered}
\mathbf{h}_{k}^{(2)}=\mathbf{h}_{k}^{(1)}-\mathbf{h}_{j}^{(1)} \mathbf{c}_{j, k} \\
\mathbf{h}_{k}^{(2)}=\mathbf{h}_{k}-\mathbf{h}_{i} \mathbf{c}_{i, k}-\left(\mathbf{h}_{j}-\mathbf{h}_{i} \mathbf{c}_{i, j}\right) \mathbf{c}_{j, k}
\end{gathered}
$$

The projection coefficient $\mathbf{c}_{j, k}$ must satisfy

$$
0 \leq \mathbf{c}_{j, k} \leq \frac{\mathbf{c}_{i, k}}{\mathbf{c}_{i, j}}
$$

If the constraint is satified, the orthogonal projection is removed. If the orthogonal projection coefficient is larger than $\mathbf{c}_{i, k} / \mathbf{c}_{i, j}$, it must be replaced by $\mathbf{c}_{i, k} / \mathbf{c}_{i, j}$, that is an oblique projection is applied. The oblique projection leads to the removal of the $1^{\text {st }}$ extreme from the $k^{\text {th }}$ row model, yielding,

$$
\mathbf{h}_{k}^{(2)}=\mathbf{h}_{k}-\mathbf{h}_{j} \frac{\mathbf{c}_{i, k}}{\mathbf{c}_{i, j}}
$$

Replacement is based on the fact that an oblique projection will decrease the residual, provided that the coefficient is bound by zero and twice the orthogonal projection coefficient. If a coefficient of a previous extreme can be driven to zero with a oblique projection with coefficient less than twice the orthogonal projection coefficient, a replacement takes place otherwise the row is not changed, as no reduction in residual is possible.

The number of extreme, non-redundant, rows will typically exceed the rank of $\mathbf{H}$. We restrict the total number of modeling vectors to this rank; once this rank is reached, a new vector is added only if the coefficient of a previous vector can be driven to zero [12]. Either the current extreme vector replaces a previous one or the row is not updated by the current vector. The selected option is the one that yields the smallest residual. The stopping criteria for the algorithm can be based on either the magnitude or the residual or an input maximum number of non-redundant inequalities to select. As the number of non-redundant inequalities will in general exceed $N$, and can be much larger, the maximum number criteria should be chosen large. If chosen too large, a number of the "non-redundant" inequalities will be nearly identical and so the output can be post-processed via clustering to further remove some redundancies. The end result is a set of extreme 


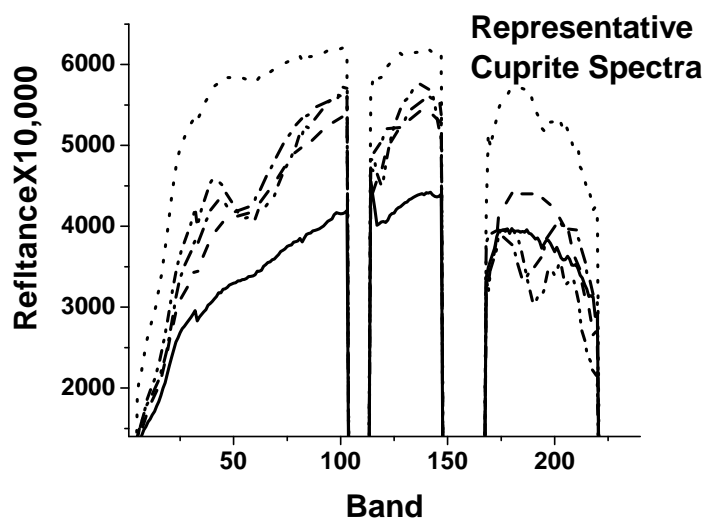

Figure 1. Representative pixel reflectance spectra selected from the Cuprite Nevada data collection, shown in the high signal to noise bands selected for processing.

rows and a set of non-negative projection coefficients that model the redundant rows as non-negative linear combinations of the extremes rows.

\subsection{Non-Negative Factorization of the Matrix}

Once rows of $\mathbf{A}$ have been identified as extreme, there remains the process of finding the non-negative expansion coefficients to complete the factorization. This is a constrained linear mixing problem. However a standard constrained least squares algorithm that requires the inverse or pseudo-inverse will not be appropriate as the number of extremes will in general exceed the pseudorank $N$ of $\mathbf{A}$. The matrix of the extremes will then be exceedingly ill-conditioned and the inverse may not exist. One approach to this problem is to select the $N$ extremes that are the most abundant, those with the most resulting non-zero projection coefficients, and perform a constrained least squares with these. The resulting residual and expansion coefficient arrays can then be further processed with the remaining extremes using the replacement procedure described above. Alternatively, the oblique projection technique [11, 12] used for the inequality constraints can be applied directly. The latter option was chosen for this work with the restriction of end-member selection to the list of columns identified as extreme by the linear inequality constraints problem. Residuals were calculated in the $\ell_{2}$ norm.

\section{Application}

The approach is applied to a spectral reflectance image data set [13] of the Cuprite Nevada mining area that was collected by the NASA Airborne Visual and InfraRed Imaging Spectrometer (AVIRIS) [14]. The AVIRIS sensor collects images in 224 contiguous bands from $400 \mathrm{~nm}$ to $2450 \mathrm{~nm}$ wavelengths. A $(200 \times 200)$ pixel subarray from the upper right hand corner of the fourth data set was selected for processing, a total of 40,000 pixel spectra. The data are expressed as reflectance times 10,000 .
We preprocessed the data removing bands 1-4, 104$113,148-167$ and 221-224 due to low signal to noise and/or strong atmospheric absorption. Sample pixel spectra are illustrated in Figure 2 and sample band images are illustrated in Figure 1. Each spectrum contained 186 high signal to noise bands. Prior estimates of the number of components from two popular new techniques $[2,3]$ lead to a range of predictions $[3,15]$ of between 14 and 25 components, even though most of SVD energy is in the 1st eight to ten singular vectors [3]. Given the wide range of estimates, we arbitrarily selected 16 as the SVD expansion length of the Cuprite array and processed the resulting linear homogeneous constraints as described above. A cutoff number of non-redundant inequalities was chosen as 40 , a number expected to exceed the number of unique band images. Many of the 40 band images were very similar. These were grouped using a simple leader clustering algorithm, QUICK [16]. The algorithm does not require the number of clusters as input, only a Euclidian distance threshold that was selected as 0.001 . The process leads to an estimate of 20 non-redundant inequalities, and 20 unique band images. The Cuprite spectral image matrix was factored using the oblique projection technique with the 20 band images. The standard deviation for the reflectance residual ranges from $0-1 \%$ of the band reflectances. The expansion coefficients are the fractional contributions of the extreme bands. These are illustrated in Figures 3, 4 and 5 . Some band-passes are modeled as scaled copies of an extreme. Examples include band-pass 5-17 (409.2$527.5 \mathrm{~nm}$ ) that is modeled by band 16 and the bandpass $132-147(1602.8-1752.2 \mathrm{~nm})$ that are modeled by band 138 as illustrated in Figures 3 and 4, respectively. Most of the bands are modeled by adjacent extremes with small contributions from more distant extremes. An exception is the band-pass 80-103 (1115.2-1314.1 nm) where the major contributions come from the local band 81 and the distant band 138, with small contributions from band 33 . Regions where strong spectral features of the minerals present [17-19] are most pronounced require several more closely spaced extreme bands, as illustrated in Figure 5.

\section{Conclusions}

The interpretation of spectral images and time series based on matrix factorization is a challenging area of research. Confounding the interpretation is the identification of the number of components present. The well known property of matrix rank provides the number of linearly independent components (rows/columns) present in the data but noise obscures the estimate of rank. The more relevant non-negative rank that provides the number of non-negative independent components present in the data is an elusive quantity that is bounded by the rank and the smaller of the number of rows/columns of the matrix. The number of extreme points in a cone also typically exceeds the rank and may provide a useful approach. The current approach, 

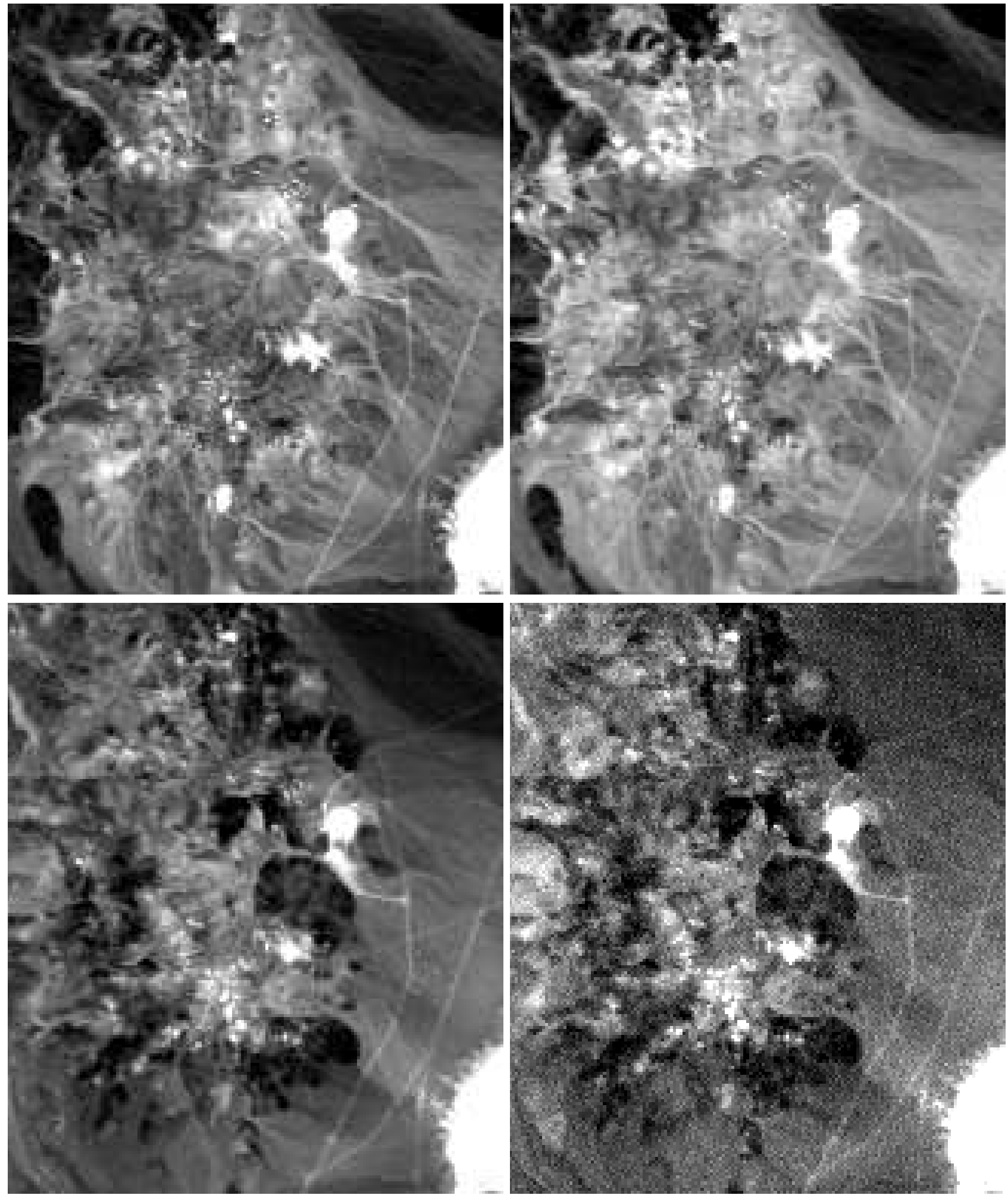

Figure 2. Four of the 224 band images of the selected upper right $(200 \times 200)$ corner of Cuprite Nevada the data collection: (Upper Left) Band 33, (664.1 nm), (Upper Right) band 138 (1662.6 nm), (Lower Left) band 189 (2158.7 nm), and (Lower Right) band 218 (2447.3 nm).

illustrated here for spectral band selection, is being extended to factorization by pixel spectra in addition to bands. The major difference is in the number of constraints. For bands the constraints number in the hundreds; for the Cuprite data, there are 224 bands; for pixel spectra however, the number of constrains range from tens of thousands to several hundred thousand; for the Cuprite data used here, there are $(200 \times 200)$ pixels and 40,000 constraints.

\section{ACKNOWLEDGMENT}

The authors would like to thank Spectral Sciences, Inc for funding the internal research and development effort that makes possible the results reported in this paper. We also greatly appreciate Dr. Raphael Panfili's assistance in the formatting this manuscript, as well as his helpful comments and suggestions. 


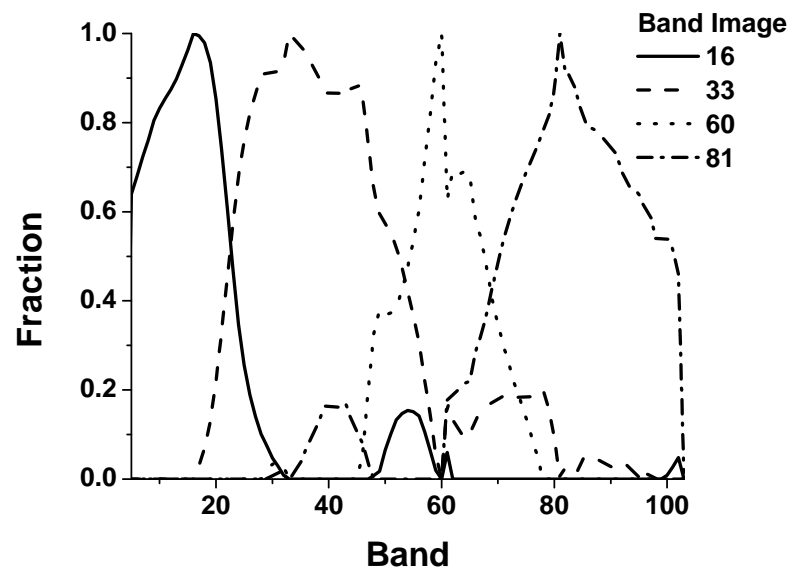

Figure 3. The fractional contributions, of the extreme band images to bands 5 to $103,(409.2-1314.1 \mathrm{~nm})$. Extreme bands $16(517.6 \mathrm{~nm}), 33$ $(664.1 \mathrm{~nm}), 60(923.0 \mathrm{~nm})$ and $81(1124.8 \mathrm{~nm})$ are illustrated.

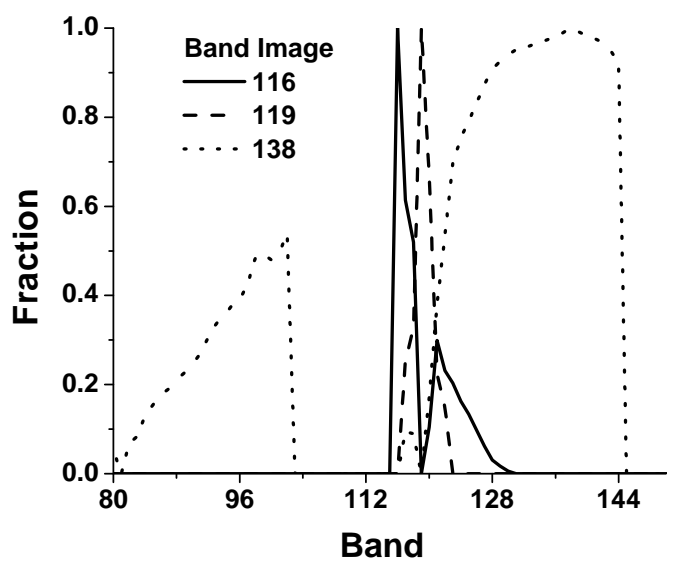

Figure 4. The fractional contributions, of the extreme band images to the high signal to noise bands 80 to $103(1115.2-1314.1 \mathrm{~nm})$ and bands 114 to 147 (1423.6-1752.2 nm). Extreme bands 116 (1443.5 nm), $119(1447.4 \mathrm{~nm})$ and $138(1662.6 \mathrm{~nm})$ are illustrated.

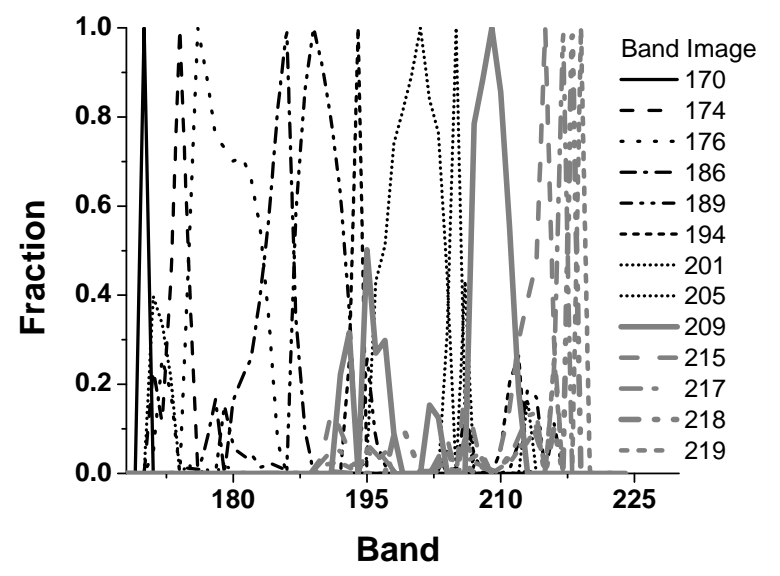

Figure 5. The fractional contributions, of the extreme band images to the high signal to noise bands 168 to $220(1948.6 \mathrm{~nm}$ to $2467.2 \mathrm{~nm})$. Extreme bands $170(1968.6 \mathrm{~nm}), 174(2008.7 \mathrm{~nm}), 176(2028.7 \mathrm{~nm}), 186$ $(2128.7 \mathrm{~nm}), 189(2158.7 \mathrm{~nm}), 194(2208.6 \mathrm{~nm}), 201(2278.4 \mathrm{~nm}), 205$ $(2318.2 \mathrm{~nm}), 209(2357.9 \mathrm{~nm}), 215(2417.6 \mathrm{~nm}), 217(2437.4 \mathrm{~nm}), 218$ $(2447.3 \mathrm{~nm})$ and $219(2457.2 \mathrm{~nm})$ are illustrated.

\section{REFERENCES}

[1] N. Acito, M. Diani, and G. Corsini, "Hyperspectral signal subspace identification in the presence of rare signal components," IEEE Transactions on Geoscience and Remote Sensing, vol. 48, no. 4, pp. 1940-1954, 2010.

[2] C.-I. Chang and Q. Du, "Estimation of number of spectrally distinct signal sources in hyperspectral imagery," IEEE Transactions on Geoscience and Remote Sensing, vol. 42, no. 3, pp. 608-619, 2004.

[3] J. M. Bioucas-Dias and J. M. Nascimento, "Hyperspectral subspace identification," IEEE Transactions on Geoscience and Remote Sensing, vol. 46, no. 8, pp. 2435-2445, 2008.

[4] A. Berman and R. J. Plemmons, "Nonnegative matrices," Classics in Applied Mathematics, SIAM, vol. 9, 1979.

[5] N. Gillis and F. Glineur, "On the geometric interpretation of the nonnegative rank," Linear Algebra and Its Applications, vol. 437, no. 11, pp. 2685-2712, 2012.

[6] J. E. Cohen and U. G. Rothblum, "Nonnegative ranks, decompositions, and factorizations of nonnegative matrices," Linear Algebra and Its Applications, vol. 190, pp. 149-168, 1993.

[7] J. Gruninger and H. Dothe, "Boundary constraints for singular value decomposition of spectral data," in SPIE Remote Sensing, vol. 8892. International Society for Optics and Photonics, 2013, pp. $88920 \mathrm{~N}-88920 \mathrm{~N}$.

[8] D. G. Luenberger, Introduction to linear and nonlinear programming. Addison-Wesley Publishing Company, 1973.

[9] R. Caron and T. Traynor, "A general framework for the analysis of sets of constraints," Mathematical Programming and Game Theory for Decision Making, p. 33, 2007.

[10] C. D. Meyer, Matrix analysis and applied linear algebra. Society for Industrial and Applied Mathematics, 2000.

[11] J. H. Gruninger, A. J. Ratkowski, and M. L. Hoke, "The sequential maximum angle convex cone (smacc) endmember model," in SPIE's Algorithms and Technologies for Multispectral, Hyperspectral, and Ultraspectral Imagery $X$, vol. 5425. International Society for Optics and Photonics, 2004, pp. 1-14.

[12] - "The extension of endmember extraction to multispectral scenes," in SPIE's Algorithms and Technologies for Multispectral, Hyperspectral, and Ultraspectral Imagery $X$, vol. 5425. International Society for Optics and Photonics, 2004, pp. 15-30.

[13] J. P. Laboratory, "Airborne visible/infrared imaging spectrometer," 2014. [Online]. Available: http://aviris. jpl.nasa.gov $/ \mathrm{html} /$ aviris.freedata.html

[14] R. O. Green, M. L. Eastwood, C. M. Sarture, T. G. Chrien, M. Aronsson, B. J. Chippendale, J. A. Faust, B. E. Pavri, C. J. Chovit, M. Solis, M. R. Olah, and O. Williams, "Imaging spectroscopy and the airborne visible/infrared imaging spectrometer (AVIRIS)," Remote Sensing of Environment, vol. 65, no. 3, pp. 227-248, 1998.

[15] T.-H. Chan, C.-Y. Chi, Y.-M. Huang, and W.-K. Ma, "A convex analysis-based minimum-volume enclosing simplex algorithm for hyperspectral unmixing," IEEE Transactions on Signal Processing, vol. 57, no. 11, pp. 44184432, 2009.

[16] J. A. Hartigan, Clustering algorithms. John Wiley \& Sons, Inc., 1975.

[17] R. N. Clark and G. A. Swayze, "Evolution in imaging spectroscopy analysis and sensor signal-to-noise: An examination of how far we have come," in Proc. 6th Annu. JPL Airborne Earth Sci. Workshop, 1996, pp. 49-53.

[18] G. A. Swayze, "The hydrothermal and structural history of the cuprite mining district, southwestern nevada: An integrated geological and geophysical approach," Ph.D. dissertation, University of Colorado at Boulder, 1997.

[19] USGS, "Cuprite, nevada research papers," 2014. [Online]. Available: http://speclab.cr.usgs.gov/cuprite.html 


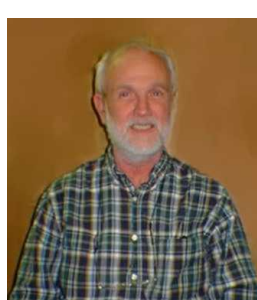

Dr. John Gruninger is a Principal Scientist at Spectral Sciences, Inc. His research interests include algorithm development for hyperspectral sensors and atmospheric radiation transport phenomena. Hyperspectral algorithm developments include constrained subspace projection techniques for detection and classification, rapid autonomous endmember selection, environmental and atmospheric effects on sub-pixel detection, and the identification and analysis of plume species. In addition he is developing techniques to fuse ultra-violet, visible and near infrared with thermal infrared data to enhance thermal atmospheric corrections, spatial resolution and material identification. Dr. Gruninger research interests also include atmospheric radiation transport. In this area, he has developed perturbation models for atmospheric structure models for gravity waves, turbulence on radiation transport, and display models for images of these structures.

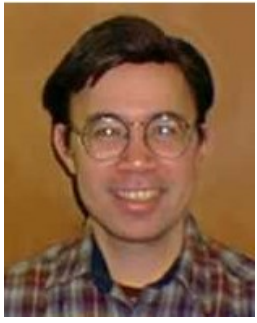

Dr. Hoang Dothe is a Principal Scientist at Spectral Sciences, Inc, and leader of the Atmospheric Backgrounds Group. He has extensive experience in atmospheric radiative transport, and models of atmospheric processes at all altitudes. His current interests include algorithms for removal of atmospheric effects, and processing image for target identification. He has developed fast computer algorithms to calculate atmospheric transmittance and radiance effects at high spectral resolution. 